\title{
1 High-titer production of aromatic amines in metabolically engineered
}

\section{Escherichia coli}

3 Taiwei Yang ${ }^{1}$, Peiling $\mathrm{Wu}^{1}$, Yang $\mathrm{Zhang}^{1}$, and Jifeng Yuan ${ }^{1, *}$

$4 \quad{ }^{1}$ State Key Laboratory of Cellular Stress Biology, Innovation Center for Cell Signaling Network,

5 School of Life Sciences, Xiamen University, Fujian 361102, China

$6 *$ Corresponding author address: School of Life Sciences, Xiamen University, Fujian 361102,

$7 \quad$ China. Email address: jfyuan@xmu.edu.cn (J Yuan)

\section{ABSTRACT}

9 Aromatic amines are widely used in the pharmaceutical industry. Here, we reported the establishment of a bacterial platform for synthesizing three types of aromatic

11 amines, namely, tyramine, dopamine, and phenylethylamine. Firstly, we expressed

12 aromatic amino acid decarboxylase from Enterococcus faecium (pheDC) in an

13 Escherichia coli strain with an increased shikimate (SHK) pathway flux toward

14 L-tyrosine or L-phenylalanine synthesis. We found that glycerol served as a better

15 carbon source than glucose, resulting in $940 \pm 46 \mathrm{mg} / \mathrm{L}$ tyramine from $4 \%$ glycerol.

16 Next, the genes of lactate dehydrogenase $(l d h A)$, formate acetyltransferase $(p f l B)$,

17 phosphate acetyltransferase (pta), and alcohol dehydrogenase ( $a d h E)$ were deleted to

18 mitigate the fermentation byproduct formation. The tyramine level was further

19 increased to $1.965 \pm 0.205 \mathrm{~g} / \mathrm{L}$ in shake flasks, corresponding to 2.1 times

20 improvement compared with that of the parental strain. By using a similar strategy,

21 we also managed to produce $703 \pm 21 \mathrm{mg} / \mathrm{L}$ dopamine and $555 \pm 50 \mathrm{mg} / \mathrm{L}$ 
22 phenethylamine. In summary, we have demonstrated that the knockout of

23 ldhA-pflB-pta-adhE is an effective strategy in improving aromatic amine productions,

24 and achieved the highest aromatic amine titers in E. coli under shake flasks reported

25 to date.

26 Key points: Aromatic amino acid decarboxylase from E. faecium was used for

27 aromatic amine production; $l d h A, p f l B$, pta together with $a d h E$ were deleted to

28 mitigate the fermentation byproduct formation; Our work represented the best

29 aromatic amine titers reported in E. coli under shake flasks.

30 Keywords: aromatic amino acid decarboxylase; tyramine; dopamine;

31 phenylethylamine; shikimate pathway; metabolic engineering

\section{Introduction}

Aromatic compounds represent a large and diverse class of chemicals that are widely used in manufacturing solvents, polymers, fine chemicals, feed and food additives, nutraceuticals, and medicines (Huccetogullari et al. 2019; Shen et al. 2020; Wang et al. 2018). Among them, aromatic amines with diverse physical characteristics are often employed as antioxidants, and precursors to pharmaceutical products (Masuo et al. 2016; Minami 2013). For example, tyramine is a high-value industrial product with widespread applications in medicine (Beltran et al. 2011). Dopamine acts as an intermediate in the biosynthesis of epinephrine and other drugs (Davie 2008; Jeong et al. 2018), and also can influence the physiological activity of plants and humans (Liang et al. 2018; Wise 2004). In addition, phenylethylamine is a precursor of antidepressants for addiction cessation (Brackins et al. 2011; Dwoskin et al. 2006). To 
44 date, the biomanufacturing process of aromatic amines mainly relies on chemical synthesis (Corrigan et al. 1945; Epstein et al. 1964). However, the chemical method typically involves complicated steps, harsh reaction conditions, and non-renewable feedstock, which is considered an environment-unfriendly process.

With the fast advancements of synthetic biology and metabolic engineering, microbial

49 synthesis of chemicals has made significant strides in recent years (Cho et al. 2015;

Huccetogullari et al. 2019). For instance, Escherichia coli has been extensively

51 utilized as the host for synthesizing many natural products, owing to its

well-characterized genetic information and abundant molecular tools (Yang et al.

2020). Aromatic amines typically use aromatic amino acids as precursors, which are

synthesized primarily through the shikimate pathway (SHK) in microorganisms

(Averesch and Kromer 2018; Shen et al. 2020). The SHK pathway is initiated by the

condensation of phosphoenolpyruvate (PEP) from the glycolytic pathway and

D-erythrose 4-phosphate $(\mathrm{E} 4 \mathrm{P})$ in the pentose phosphate $(\mathrm{PP})$ pathway to produce

3-deoxy-D-arabino-heptulosonate-7-phosphate (DAHP), which is subsequently

transformed to aromatic amino acids (Averesch and Kromer 2018; Cao et al. 2020)

(Fig. 1). Koma et al. overexpressed a cluster of genes from the SHK pathway and a

61 heterologous decarboxylase gene in E. coli, and the resulting strain produced $6.3 \mathrm{mM}$

(863mg/L) tyramine (Koma et al. 2012). Heterologous expression of tyrosinase and

63 decarboxylase in the L-tyrosine overproducing E. coli resulted in $260 \mathrm{mg} / \mathrm{L}$ dopamine

64 (Nakagawa et al. 2011).

In this work, we aimed to develop an E. coli platform to improve the biosynthesis of 
66

aromatic amines. As shown in Fig. 1, the main strategy used to improve aromatic amines production comprises the deletion of fermentation byproduct-related pathways to enhance the metabolic flux of the SHK pathway. In particular, we investigated the effect on aromatic amine biosynthesis by knockout of lactate dehydrogenase (ldhA), formate acetyltransferase $(p f l B)$, phosphate acetyltransferase (pta), and alcohol dehydrogenase (adhE).

\section{Materials and methods}

\section{Strains and reagents}

E. coli $\mathrm{DH} 5 \alpha$ was utilized to construct plasmids, and E. coli MG1655 (DE3) derived strain with $\Delta$ tyrA and $\Delta$ pheA (Lai et al. 2022) was employed as the chassis cell for aromatic amine production. LB medium containing $10 \mathrm{~g} / \mathrm{L}$ tryptone, $5 \mathrm{~g} / \mathrm{L}$ yeast extract, and $10 \mathrm{~g} / \mathrm{L} \mathrm{NaCl}$ was applied for cultivating E. coli. Appropriate antibiotics (100 $\mu \mathrm{g} / \mathrm{mL}$ ampicillin, $50 \mu \mathrm{g} / \mathrm{mL}$ kanamycin, $34 \mu \mathrm{g} / \mathrm{mL}$ chloramphenicol) were supplemented to maintain the plasmids when needed. Enzymes (high-fidelity phusion polymerase, BamHI-HF, XhoI, BsaI-HF, Esp3I-HF, and T4 DNA ligase) were purchased from New England Biolabs (Beverly, MA, USA). PCR purification kit, gel extraction kit, and plasmid DNA extraction kit were all purchased from BioFlux (Shanghai, China). The details of chemicals used in this study are provided in Supplementary materials.

\section{Plasmid and strain construction}

All the genes were PCR amplified using high-fidelity phusion polymerase. The oligonucleotides used in this study are listed in Supplementary Table S1. The gene 
encoding pheDC from E. faecium (Genbank: AJ783966.1) was codon optimized for $E$. coli and synthesized by GenScript (Nanjing, China). The genes encoding hpaBC (Genbank: Z37980) were obtained from the genomic DNA of E. coli.

The plasmid pET-AroG ${ }^{\mathrm{fbr}}-{ }_{-} \mathrm{Tyr} \mathrm{A}^{\mathrm{fbr}}$ and pET-AroG $^{\mathrm{fbr}}-\mathrm{PheA}^{\mathrm{fbr}}$ were constructed in a similar way to our previous report (Lai et al. 2022). In brief, the genes encoding $t y r A^{f b r}$, $p h e A^{f b r}$, and $a r o G^{f b r}$ were obtained from the genomic DNA of E. coli via overlapping PCR amplification process, digested with Esp3I, and ligated into pETDuet-1 between BamHI and XhoI sites. For the plasmid pRSF-PheDC, the synthesized pheDC gene was inserted into pRSFDuet-1 between BamHI and XhoI sites. For the plasmid pACYC-HpaBC, the $h p a B C$ gene was amplified using E. coli genomic DNA as a template, and inserted into pACYCDuet-1 between BamHI and XhoI sites. For constructing MG1655 (DE3) derived $\Delta$ pflB- $\Delta$ ldhA- $\Delta$ pta- $\Delta$ adhE strain, the gene knockout procedure was carried out via the CRISPR/Cas9 method (Cong et al. 2013). The engineering strains with corresponding plasmids were obtained by standard electroporation or heat-shock approach. All the details of plasmids and strains are provided in Supplementary Table S2.

\section{Shake flask cultivation}

Colonies were inoculated from solid agar plates into $10 \mathrm{~mL}$ test tubes containing 2 $\mathrm{mL} \mathrm{LB}$ medium and cultivated at $37^{\circ} \mathrm{C}$ and $250 \square \mathrm{rpm}$ to prepare the seed culture. The next day, fresh overnight cultures $(0.15 \mathrm{~mL})$ were inoculated into $50 \mathrm{~mL}$ shake flasks containing $15 \mathrm{~mL}$ modified M9 medium with appropriate antibiotics. The components of the modified M9 medium were given in Supplementary Material. Upon the cell 
110 density reaching $0.4-0.6$, isopropyl $\beta$-D-1-thiogalactopyranoside (IPTG) was added to

111 the media to a final concentration of $10 \mu \mathrm{M}$ for inducing the gene expressions. The

112 cell cultures were shifted to $30^{\circ} \mathrm{C}$ and $250 \square \mathrm{rpm}$ for the aromatic amine productions.

113 Samples were periodically taken for monitoring the cell growth by using a microplate

114 reader (Biotek, Synergy H1).

\section{HPLC analysis of aromatic amine levels}

116 The samples were centrifuged at $14,000 \square \mathrm{rpm}$ for $10 \square \mathrm{min}$ to remove the cellular

117 pellets. Shimadzu LC-20A system equipped with a photodiode array detector and a

118 reversed-phase C18 column $(150 \mathrm{~mm} \times 4.6 \mathrm{~mm} \times 5 \mu \mathrm{m})$ was used for the quantitation

119 of aromatic amines. The column was maintained at a temperature of $40{ }^{\circ} \mathrm{C}$. To

120 identify tyramine and phenethylamine, the mobile phase comprising $90 \%$ ultrapure

$121 \mathrm{H}_{2} \mathrm{O}$ (supplemented with $0.1 \%$ trifluoroacetic acid) and $10 \%$ acetonitrile was used.

122 For dopamine detection, the mobile phase containing 95\% ultrapure $\mathrm{H}_{2} \mathrm{O}$

123 (supplemented with $0.1 \%$ trifluoroacetic acid) and 5\% acetonitrile was used. The flow

124 rate was maintained at $1.0 \mathrm{~mL} / \mathrm{min}$. The wavelengths used for detecting tyramine,

125 dopamine and phenethylamine were set at $222 \mathrm{~nm}, 203 \mathrm{~nm}$, and $208 \mathrm{~nm}$, respectively.

126 The retention times of tyramine, dopamine and phenethylamine were $3.5 \mathrm{~min}, 3.8 \mathrm{~min}$

127 and $8.3 \square \min$, respectively. The aromatic amine levels were quantitated using an

128 external standard curve based on authentic standards.

\section{Results}

\section{De novo production of tyramine in $E$. coli}

131 As shown in Fig.1, aromatic amines such as tyramine and phenethylamine can be 
132 produced by coupling the aromatic amino acid synthesis with heterologous expression

133 of aromatic amino acid decarboxylase (AADC). In this study, we chose AADC from

134 E. faecium (PheDC) as it has been functionally expressed in E. coli, resulting in

135 L-phenylalanine and L-tyrosine decarboxylase activities (Marcobal et al. 2006). To

136 increase the metabolic flux toward the SHK pathway, the feedback-resistant genes

137 encoding 3-deoxy-D-arabinoheptulosonate-7-phosphate synthase (aroG) together

138 with chorismate mutase/prephenate dehydratase (pheA) or chorismate

139 mutase/prephenate dehydrogenase (tyrA) were overexpressed in MG1655 (DE3) with

$140 \Delta$ tyrA $\Delta$ pheA. As shown in Fig.2a, we constructed two plasmids for expressing

$141 \quad a r o G^{f b r}, t y r A^{f b r}$, and pheDC for tyramine production in E. coli. To identify suitable

142 carbon sources for the synthesis of aromatic amines, we compared the modified M9

143 media with $4 \%(\mathrm{w} / \mathrm{v})$ glycerol or glucose for tyramine productions. As shown in Fig.

$1442 \mathrm{~b}$, the tyramine titer reached $940 \pm 46 \mathrm{mg} / \mathrm{L}$ when $4 \%$ glycerol was used, whereas

145 only $656 \pm 72 \mathrm{mg} / \mathrm{L}$ tyramine was obtained in glucose-containing medium. The

146 maximum levels of tyrpine were achieved around $36 \mathrm{~h}$, and further cultivation did not

147 obviously improve the titer.

148 Tyramine production by abolishing fermentation side-pathways

149 When cultured in the absence of oxygen, E. coli undergoes a mixed acid fermentation

150 (Forster and Gescher 2014), resulting in the production of ethanol, acetate, lactate,

151 and formate (Clark 1989; Gonzalez et al. 2008). These fermentation byproducts are

152 respectively mediated by lactate dehydrogenase (idhA) and formate acetyltransferase

$153(p f l B)$ in the pyruvate catabolism and by phosphate acetyltransferase $(p t a)$ and alcohol 
Even under aerobic circumstances, E. coli diverts a significant amount of carbon flow

to fermentation byproducts as a consequence of glycolytic overflow (Kang et al. 2009). It was reported that the accumulation of by-products such as lactate, acetate, and formate would arrest the cell growth (Causey et al. 2004). In addition, James Liao's group has demonstrated that the knockout of genes that contribute to the fermentation byproduct formation such as adhE, ldhA, pflB, and pta could substantially improve isobutanol productions in E. coli (Atsumi et al. 2008).

Therefore, the reduced formation of fermentation byproducts would theoretically channel more carbon flux to the products-of-interest, and maximize the productivity. In this study, we further proceeded to engineer the $E$. coli metabolism by mitigating byproduct formations, so that more metabolic flux could be diverted to aromatic amines. In particular, lactate dehydrogenase $(l d h A)$, formate acetyltransferase $(p f l B)$, phosphate acetyltransferase (pta), and alcohol dehydrogenase ( $a d h E)$ were deleted by CRISPR/Cas9 mediated approach (Fig. 3a). According to Fig. 3b, the deletions of side pathway genes resulted in a slightly slower growth rate of strain TA3.0 than that of TA1.0 at the initial stage, indicating that disruption of fermentation related pathways would slightly affect the cells growth at the initial phase. However, strain TA3.0 surpassed the growth of strain TA1.0 after $12 \mathrm{~h}$, probably because more carbon flux toward the TCA cycle enhances energy utilization, resulting in a higher cell density at the later phase. As shown in Fig. 3b, strain TA3.0 produced 1.965 \pm 0.205 g/L tyramine 
confirming that disruption of fermentation related pathways could effectively increase more carbon flux toward aromatic amine synthesis.

\section{Dopamine production using the strains carrying the $h p a B C$ gene}

For investigating the feasibility of this platform for producing other aromatic amines,

we continued our efforts to synthesize dopamine, an important pharmaceutical

compound. Endogenous 4-hydroxyphenylacetate 3-monooxygenase from E. coli is an enzyme with two components encoded by $h p a B$ and $h p a C$ genes that adds a second hydroxyl group at the ortho position to 4-hydroxyphenylacetate and L-tyrosine (Guo et al. 2021). As shown in Fig. 4a, L-tyrosine can be hydroxylated to form dopamine. Alternatively, the hydroxylation step might also occur at 4-hydroxyphenylacetate or tyramine. Overall, the enzyme cascade for dopamine production comprises AroG ${ }^{\mathrm{fbr}}$, TyrA ${ }^{\mathrm{fbr}}, \mathrm{HpaBC}$, and PheDC (Fig. 4b). As shown in

Fig. 4c, there was no significant difference in cell growth between strain DA1.0 and

DA3.0 during dopamine synthesis. Notably, strain DA3.0 exhibited the highest level of dopamine production $(703 \pm 21 \mathrm{mg} / \mathrm{L})$ at $48 \mathrm{~h}$, which is nearly 2.9 times than that of stain DA1.0 (242 $\pm 24 \mathrm{mg} / \mathrm{L})$. Therefore, we achieved a considerable improvement in dopamine production than the previous study (Nakagawa et al. 2011).

As mentioned above, L-tyrosine can also be first converted to tyramine under the action of PheDC (Fig. 4a). To this end, we also measured the accumulation of 
toward tyramine hydroxylation might be not sufficient. Surprisingly, more tyramine was accumulated in the DA1.0 strain than that of strain DA3.0. We reasoned that the hydroxylation reaction requires a large amount of $\mathrm{NADH}$ and $\mathrm{NADPH}$, critical cofactors for the flavin reduction by hpaC. Therefore, knockout of side-pathways that consume NADH and NADPH might favor the hydroxylation reaction with improved dopamine production in strain DA3.0.

\section{Phenylethylamine production using the engineered $E$. coli strain}

Next, we also proceeded with phenylethylamine production in the engineered E. coli. Briefly, we replaced $a r o G^{f b r}-t y r A^{f b r}$ plasmid with $a r o G^{f b r}-p h e A^{f b r}$ to switch the metabolic flux from L-tyrosine to L-phenylalanine synthesis. The two-plasmid system with enzyme cascade including $a r o G^{f b r}-p h e A^{f b r}$ and $p h e D C$ was used to convert L-phenylalanine to phenylethylamine (Fig. 5a). As depicted in Fig. 5b, the maximum phenethylamine production of $555 \pm 50 \mathrm{mg} / \mathrm{L}$ was achieved by strain PEA3.0 after $72 \mathrm{~h}$, which was increased by 2.28 -fold compared with that of strain PEA1.0 $(169 \pm 20$ $\mathrm{mg} / \mathrm{L})$. The phenethylamine level achieved in the shake flask by our study was also much higher than that of a recent report (Xu and Zhang 2020). In addition, we found that both strain PEA1.0 and PEA3.0 gave a similar growth profile (Fig. 5b). However, the final cell densities for phenylethylamine-producing strains were slightly lower than dopamine or tyramine-producing strains. Since strain PEA1.0 and 3.0 with different levels of phenylethylamine gave a similar growth rate, we concluded that phenylethylamine is not toxic to the cells at the current concentrations. Therefore, it is likely that the reduced biomass of phenylethylamine-producing strains was mainly 
caused by $\triangle t y r A$.

221

222

\section{Discussion}

Aromatic amines have a wide range of applications in medicine, chemistry, and biology. Rapid advances in synthetic biology have facilitated the study of aromatic compound biosynthesis and offered an engineering framework for producing high-value aromatic compounds. E. coli has been genetically engineered to boost the productivity of aromatic compounds by removing or inhibiting undesirable genes and increasing the expression of rate-limiting genes. In this study, we applied similar strategies to increase the metabolic flux toward the SHK pathway. By introducing feedback-resistant versions of $t y r A^{f b r} / p h e A^{f b r}$ and $a r o G^{f b r}$, the recombinant strains with a further expression of pheDC from E. faecium were able to produce $940 \pm 46 \mathrm{mg} / \mathrm{L}$ tyramine, $242 \pm 24 \mathrm{mg} / \mathrm{L}$ dopamine, and $169 \pm 20 \mathrm{mg} / \mathrm{L}$ phenylethylamine, respectively. According to recent studies, decreasing mixed acid fermentation is proved to be effective in increasing the production of 3-hydroxypropionate (3HP) (Liu et al. 2018), polyhydroxyalkanoate (Jung et al. 2019), $\beta$-alanine (Zou et al. 2020), and 2,3-butanediol (2,3-BD) (Song et al. 2019). Therefore, we further constructed a high titer aromatic amine production platform by abolishing fermentation side-pathways. Namely, the genes of (i) $l d h A$ and $p f l B$ (from pyruvate to lactate and formate) and (ii) pta and $a d h E$ (from acetyl-CoA to acetate and ethanol) were deleted to improve the metabolic flux from the carbon source to the SHK pathway. Finally, $1.965 \pm 0.205 \mathrm{~g} / \mathrm{L}$ tyramine, $703 \pm 21 \mathrm{mg} / \mathrm{L}$ dopamine, and $555 \pm 50 \mathrm{mg} / \mathrm{L}$ phenethylamine were obtained from $40 \mathrm{~g} / \mathrm{L}$ glycerol in shake flask cultivation. The titers achieved by us were much 
242 higher than previous reports under shake flask conditions (Koma et al. 2012;

243 Nakagawa et al. 2011; Xu and Zhang 2020).

244 During the dopamine biosynthesis, we observed that both strain DA1.0 and DA3.0

245 accumulated a substantial amount of tyramine, suggesting that the HpaBC could not

246 effectively hydroxylate tyramine to dopamine. Considering that a mutant HpaBC was

247 recently identified with good activity toward the tyramine hydroxylation (Chen et al.

248 2019), it will be possible to address tyramine accumulation by simply introducing the

249 mutant $\mathrm{HpaBC}$ to our engineered E. coli strain. In addition, we were surprised to find

250 out that less tyramine was accumulated in strain DA3.0 when compared to that of

251 strain DA1.0. Since the hydroxylation reaction requires additional reducing power for

252 flavin recycling, the knockout of fermentation side-pathways that consume NADH

253 and NADPH would also improve dopamine synthesis. Therefore, it is likely that our

254 engineered platform would be of great interest for hosting other biosynthetic

255 pathways that require cofactors such as NADH and NADPH.

256 In summary, we have optimized the SHK pathway and eliminated the side-pathways

257 involved in the mixed acid fermentation to enable high-titer generation of aromatic

258 amines in metabolically modified E. coli. We demonstrated that the knockout of

$259 l d h A-p f l B-p t a-a d h E$ is an effective strategy in improving aromatic amine productions.

260 Based on our findings, we believe that the E. coli system has great prospects for the

261 future industrial-scale aromatic amine production. Moreover, these engineered strains

262 might also be used to manufacture other aromatic compounds with pharmaceutical

263 value. 


\section{Author contributions}

J. Y. conceived and designed the project. T. Y. and P. W. constructed the plasmids, strains and collected the data. T. Y. and Y. Z. analyzed the date. T.Y., Y. Z. and J. Y. wrote the manuscript.

\section{Funding information}

We acknowledge financial support from Xiamen University under grant no. 0660-X2123310 and ZhenSheng Biotech, China.

\section{Compliance with ethical standards}

Conflicts of interest The authors declare that they have no competing interests.

Ethical approval This study does not contain any studies with human participants or animals performed by any of the authors.

Data availability All data generated or analyzed during this study are included in this published article [and its supplementary information files].

\section{References}

Atsumi S, Hanai T, Liao JC (2008) Non-fermentative pathways for synthesis of branched-chain higher alcohols as biofuels. Nature 451(7174):86-9 doi:10.1038/nature06450

Averesch NJH, Kromer JO (2018) Metabolic Engineering of the Shikimate Pathway for Production of Aromatics and Derived Compounds-Present and Future Strain Construction Strategies. Front Bioeng Biotech 6 doi:10.3389/fbioe.2018.00032

Beltran B, Carrillo R, Martin T, Martin VS, Machado JD, Borges R (2011) Fluorescent beta-Blockers as Tools to Study Presynaptic Mechanisms of Neurosecretion. Pharmaceuticals (Basel) 4(5):713-25 doi:10.3390/ph4050713 
286 Brackins T, Brahm NC, Kissack JC (2011) Treatments for methamphetamine abuse: a 287 literature review for the clinician. J Pharm Pract 24(6):541-50 doi: $10.1177 / 0897190011426557$

Cao MF, Gao MR, Suastegui M, Mei YZ, Shao ZY (2020) Building microbial factories for the production of aromatic amino acid pathway derivatives: From commodity chemicals to plant-sourced natural products. Metab 58:94-132 doi:10.1016/j.ymben.2019.08.008

Causey TB, Shanmugam KT, Yomano LP, Ingram LO (2004) Engineering Escherichia colifor efficient conversion of glucose to pyruvate. P Natl Acad Sci USA 101(8):2235-2240 doi:10.1073/pnas.0308171100

Chen W, Yao J, Meng J, Han WJ, Tao Y, Chen YH, Guo YX, Shi GZ, He Y, Jin JM, Tang SY (2019) Promiscuous enzymatic activity-aided multiple-pathway network design for metabolic flux rearrangement in hydroxytyrosol biosynthesis. Nat Commun 10 doi:10.1038/s41467-019-08781-2

Cho C, Choi SY, Luo ZW, Lee SY (2015) Recent advances in microbial production of fuels and chemicals using tools and strategies of systems metabolic engineering. Biotechnol Adv 33(7):1455-1466 doi:10.1016/j. biotechadv.2014.11.006

Clark DP (1989) The fermentation pathways of Escherichia coli. FEMS Microbiol Rev 5(3):223-34 doi:10.1016/0168-6445(89)90033-8

Cong L, Ran FA, Cox D, Lin S, Barretto R, Habib N, Hsu PD, Wu X, Jiang W, Marraffini LA, Zhang F (2013) Multiplex genome engineering using CRISPR/Cas systems. Science 339(6121):819-23 doi: 10.1126/science. 1231143 
308 Corrigan JR, Langerman M-J, Moore ML (1945) Preparation of N-Substituted

309 1-(p-Hydroxyphenyl)-2-aminoethanols. J Am Chem Soc 67(11):1894-1896

$310 \quad$ doi:10.1021/ja01227a004

311 Davie CA (2008) A review of Parkinson's disease. Brit Med Bull 86(1):109-127

312 doi:10.1093/bmb/ldn013

313 Dwoskin LP, Rauhut AS, King-Pospisil KA, Bardo MT (2006) Review of the pharmacology and

314 clinical profile of bupropion, an antidepressant and tobacco use cessation agent. Cns

315 Drug Rev 12(3-4):178-207 doi:10.1111/j.1527-3458.2006.00178.x

316 Epstein J, Michel HO, Rosenblatt DH, Plapinger RE, Stephani RA, Cook E (1964) Reactions of

317 Isopropyl Methylphosphonofluoridate with Substituted Phenols. II. J Am Chem Soc

318 86(22):4959-4963 doi: $10.1021 / j a 01076 a 043$

319 Forster AH, Gescher J (2014) Metabolic Engineering of Escherichia coli for Production of

320 Mixed-Acid Fermentation End Products. Front Bioeng Biotechnol 2:16

Gonzalez R, Murarka A, Dharmadi Y, Yazdani SS (2008) A new model for the anaerobic fermentation of glycerol in enteric bacteria: trunk and auxiliary pathways in Escherichia coli. Metab Eng 10(5):234-45 doi:10.1016/j.ymben.2008.05.001

Guo DY, Kong SJ, Sun Y, Li X, Pan H (2021) Development of an artificial biosynthetic pathway for biosynthesis of (S)-reticuline based on HpaBC in engineered Escherichia coli. Biotechnol Bioeng doi:10.1002/bit.27924

Huccetogullari D, Luo ZW, Lee SY (2019) Metabolic engineering of microorganisms for 
doi:10.1186/s12934-019-1090-4

331
Jeong EH, Sunwoo MK, Song YS (2018) Serial I-123-FP-CIT SPECT Image Findings of Parkinson's Disease Patients With Levodopa-Induced Dyskinesia. Front Neurol 9:1133 doi:10.3389/fneur.2018.01133

Jung HR, Yang SY, Moon YM, Choi TR, Song HS, Bhatia SK, Gurav R, Kim EJ, Kim BG, Yang YH (2019) Construction of Efficient Platform Escherichia coli Strains for Polyhydroxyalkanoate Production by Engineering Branched Pathway. Polymers (Basel) 11(3) doi:10.3390/polym11030509

Kang Z, Geng Y, Xia Y, Kang J, Qi Q (2009) Engineering Escherichia coli for an efficient $\begin{array}{llll}\text { aerobic fermentation } & \text { platform. } \mathrm{J} \text { Biotechnol 144(1):58-63 }\end{array}$ doi:10.1016/j.jbiotec.2009.06.021

Koma D, Yamanaka H, Moriyoshi K, Ohmoto T, Sakai K (2012) A convenient method for multiple insertions of desired genes into target loci on the Escherichia coli chromosome. Appl Microbiol Biotchnol 93(2):815-829 doi:10.1007/s00253-011-3735-z

Lai Y, Chen H, Liu L, Fu B, Wu P, Li W, Hu J, Yuan J (2022) Engineering a Synthetic Pathway for Tyrosol Synthesis in Escherichia coli. Acs Synth Biol doi:10.1021/acssynbio. 1c00517

Liang B, Gao T, Zhao Q, Ma C, Chen Q, Wei Z, Li C, Li C, Ma F (2018) Effects of Exogenous Dopamine on the Uptake, Transport, and Resorption of Apple lonome Under Moderate Drought. Front Plant Sci 9:755 doi:10.3389/fpls.2018.00755

Liu R, Liang L, Choudhury A, Bassalo MC, Garst AD, Tarasava K, Gill RT (2018) Iterative genome editing of Escherichia coli for 3-hydroxypropionic acid production. Metab Eng 
Marcobal A, de las Rivas B, Munoz R (2006) First genetic characterization of a bacterial

Nakagawa A, Minami H, Kim JS, Koyanagi T, Katayama T, Sato F, Kumagai H (2011) A bacterial platform for fermentative production of plant alkaloids. Nat Commun 2:326 doi:10.1038/ncomms 1327

Shen YP, Niu FX, Yan ZB, Fong LS, Huang YB, Liu JZ (2020) Recent Advances in Metabolically Engineered Microorganisms for the Production of Aromatic Chemicals Derived From Aromatic Amino Acids. Front Bioeng Biotech 8:407 doi: $10.3389 /$ fbioe. 2020.00407

Song CW, Park JM, Chung SC, Lee SY, Song H (2019) Microbial production of 2,3-butanediol for industrial applications. J Ind Microbiol Biotechnol 46(11):1583-1601 doi:10.1007/s10295-019-02231-0

Trotter EW, Rolfe MD, Hounslow AM, Craven CJ, Williamson MP, Sanguinetti G, Poole RK, Green J (2011) Reprogramming of Escherichia coliK-12 Metabolism during the Initial Phase of Transition from an Anaerobic to a Micro-Aerobic Environment. Plos One 6(9):e25501 doi:10.1371/journal.pone.0025501 
374 Wang J, Shen XL, Rey J, Yuan QP, Yan YJ (2018) Recent advances in microbial production of aromatic natural products and their derivatives. Appl Microbiol Biotechnol

Wise RA (2004) Dopamine, learning and motivation. Nat Rev Neurosci 5(6):483-94 doi: $10.1038 / \mathrm{nrn} 1406$

Xu DQ, Zhang LR (2020) Pathway Engineering for Phenethylamine Production in Escherichia coli. J Agr Food Chem 68(21):5917-5926 doi:10.1021/acs.jafc.0c01706

Yang D, Park SY, Park YS, Eun H, Lee SY (2020) Metabolic Engineering of Escherichia coli (2020) Pathway construction and metabolic engineering for fermentative production of beta-alanine in Escherichia coli. Appl Microbiol Biotechnol 104(6):2545-2559 


\section{Figure Captions}

396 Fig. 1. Schematic diagram of aromatic amine production from the shikimate (SHK)

397 pathway. PP pathway: pentose phosphate pathway; AroG,

3-deoxy-D-arabinoheptulosonate-7-phosphate synthase; TyrA, chorismate

mutase/prephenate dehydrogenase; PheA, chorismate mutase/prephenate dehydratase;

HapBC, 4-hydroxyphenylacetate 3-monooxygenase; AADC, aromatic amino acid

decarboxylase. DHAP: Dihydroxyacetone phosphate; E4P: D-erythrose 4-phosphate;

PEP: Phosphoenolpyruvate; DAHP: 3-deoxy-D-arabino-heptulosonate-7-phosphate;

CHOR: Chorismate; PREPH: Prephenate; L-Tyr, L-tyrosine; L-Phe, L-phenylalanine.

$l d h A$ encodes D-lactate dehydrogenase; $p f l B$ encodes pyruvate formate lyase; pta

highlights the fermentaion byproduct related pathways. Dashed lines illustrate

multiple steps.

Fig. 2. Engineering E. coli for tyramine overproduction. (a) The plasmids for

tyramine

production.

AroG $G^{f b r}$,

feedback

resistant

410 3-deoxy-d-arabinoheptulosonate-7-phosphate synthase; TyrA ${ }^{f b r}$, feedback resistant

411 chorismate mutase/prephenate dehydrogenase; PheDC, aromatic amino acid

412 decarboxylase from E. faecium. (b) Growth profile and tyramine production of strain 
413 TA1.0 under shake flasks. All the experiments were carried out in $15 \mathrm{~mL}$ modified

414 M9 medium containing $40 \mathrm{~g} / \mathrm{L}$ glycerol or $40 \mathrm{~g} / \mathrm{L}$ glucose. Experiments were

415 performed in triplicate biological repeats and the data represent the mean value with

416 standard deviation.

417 Fig. 3. Knockout of fermentation byproduct related pathways substantially improved

418 tyramine production. (a) Agarose gel image for PCR verification of gene knockout

419 events. Strain G1.0 strain with $\Delta$ tyrA- $\Delta$ pheA was used as the control. Strain G3.0 with

420 further deletion of $p f l B, l d h A, a d h E$, and $p t a$ was confirmed by diagnostic PCR. (b)

421 Growth profile and tyramine production of strains TA1.0 and TA3.0. All the

422 experiments were carried out in $15 \mathrm{~mL}$ modified M9 medium containing $40 \mathrm{~g} / \mathrm{L}$

423 glycerol. Experiments were performed in triplicate biological repeats and the data

424 represent the mean value with standard deviation.

425 Fig. 4. The dopamine production in shake flasks. (a) The proposed biosynthetic route

426 toward dopamine synthesis. The dashed arrow of HpaBC indicates the poor activity of

$427 \mathrm{HpaBC}$ in converting tyramine to dopamine. (b) The plasmids for dopamine

428 production. (c) Growth profile and dopamine production of strains DA1.0 and DA3.0.

429 (d) Tyramine accumulation in strains DA1.0 and DA3.0. All the experiments were

430 carried out in $15 \mathrm{~mL}$ modified M9 medium containing $40 \mathrm{~g} / \mathrm{L}$ glycerol. Experiments 
431 were performed in triplicate biological repeats and the data represent the mean value

432 with standard deviation.

433 Fig. 5. The phenethylamine production in shake flasks. (a) The plasmids for

434 phenethylamine production. PheA ${ }^{\mathrm{fbr}}$, feedback-resistant chorismate

435 mutase/prephenate dehydratase. (b) Growth profile and phenethylamine production of

436 strains PEA1.0 and PEA3.0. All the experiments were carried out in $15 \mathrm{~mL}$ of

437 modified M9 medium containing $40 \mathrm{~g} / \mathrm{L}$ glycerol. Experiments were performed in

438 triplicate biological repeats and the data represent the mean value with standard

439 deviation.

440 


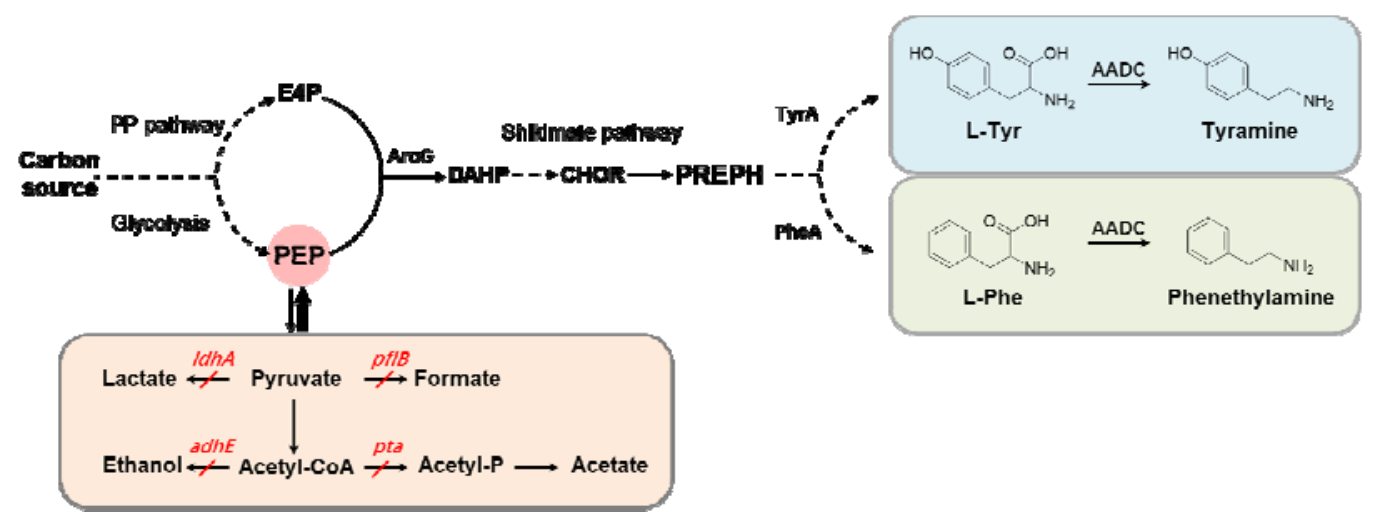

444 Fig. 1. 
a

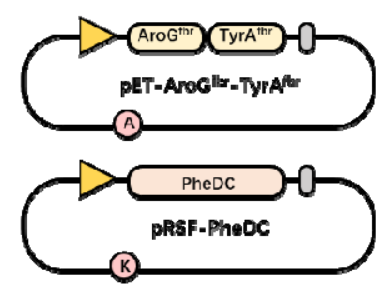

447

448

449

Fig. 2. b

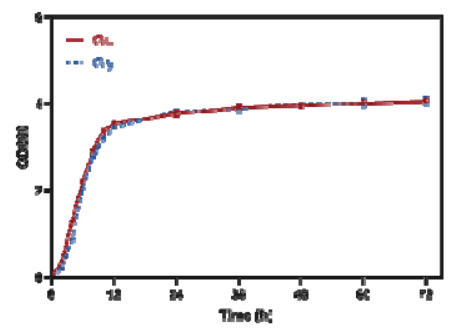

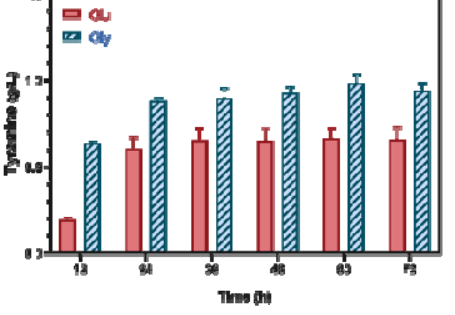


bioRxiv preprint doi: https://doi.org/10.1101/2022.01.14.476304; this version posted January 14,2022 . The copyright holder for this preprint (which was not certified by peer review) is the author/funder. All rights reserved. No reuse allowed without permission.

450

a

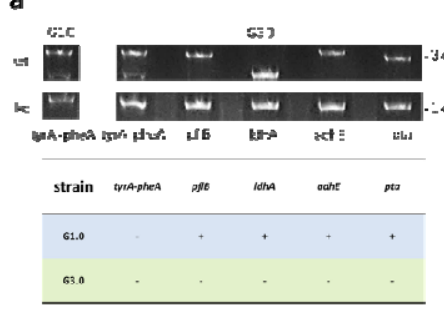

b

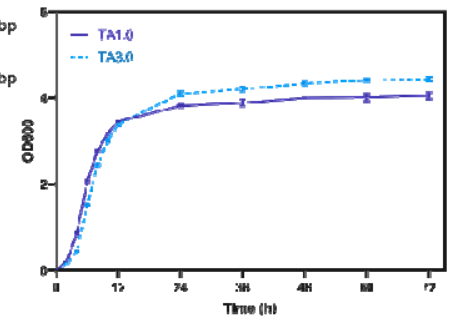

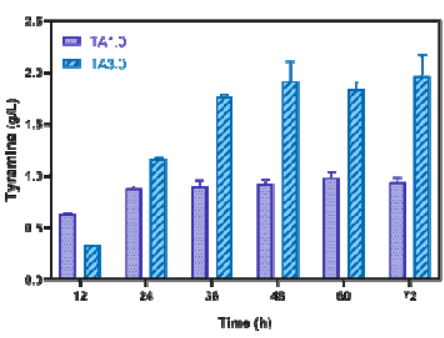

451 Fig. 3.

452 
a

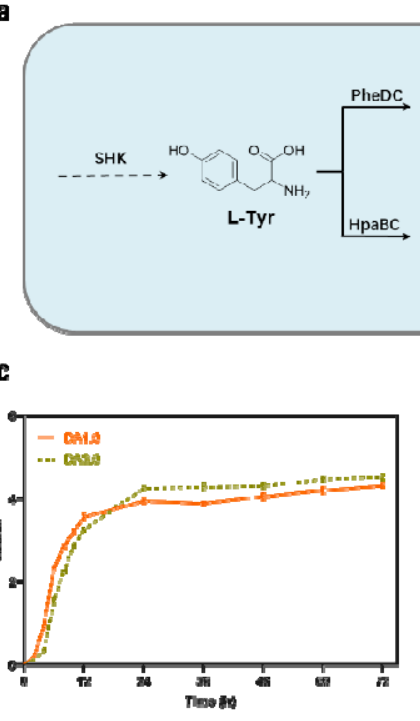

454

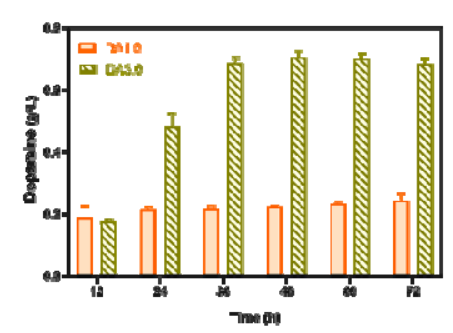

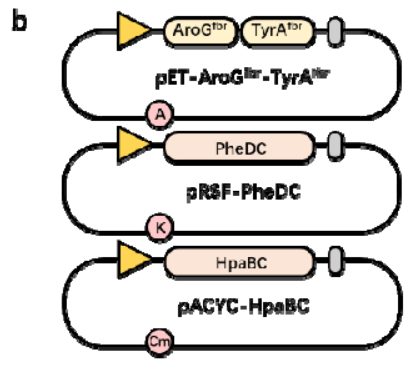

d

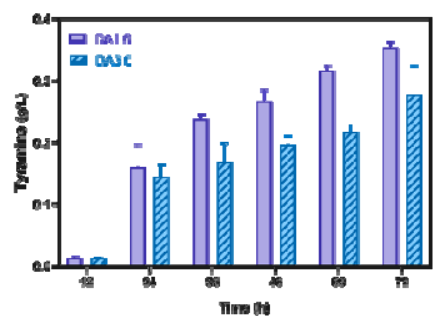

455

Fig. 4.

456 
bioRxiv preprint doi: https://doi.org/10.1101/2022.01.14.476304; this version posted January 14,2022 . The copyright holder for this preprint (which was not certified by peer review) is the author/funder. All rights reserved. No reuse allowed without permission.

a

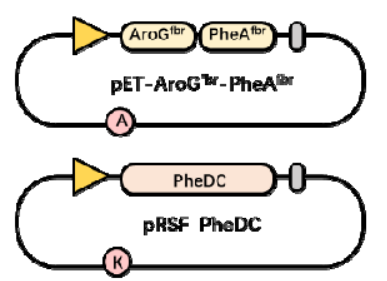

459

460

461

462

Fig. 5. b
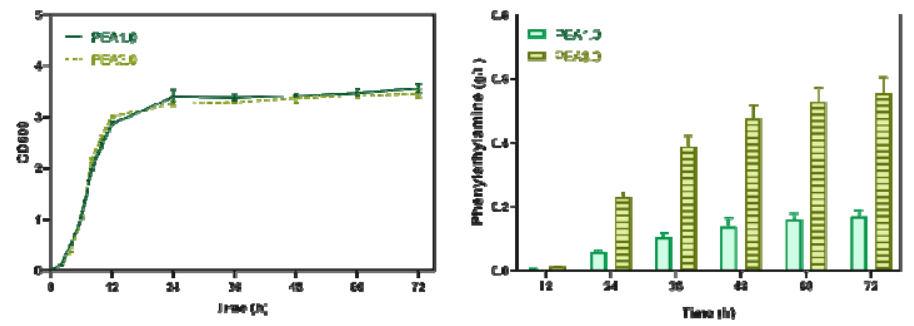


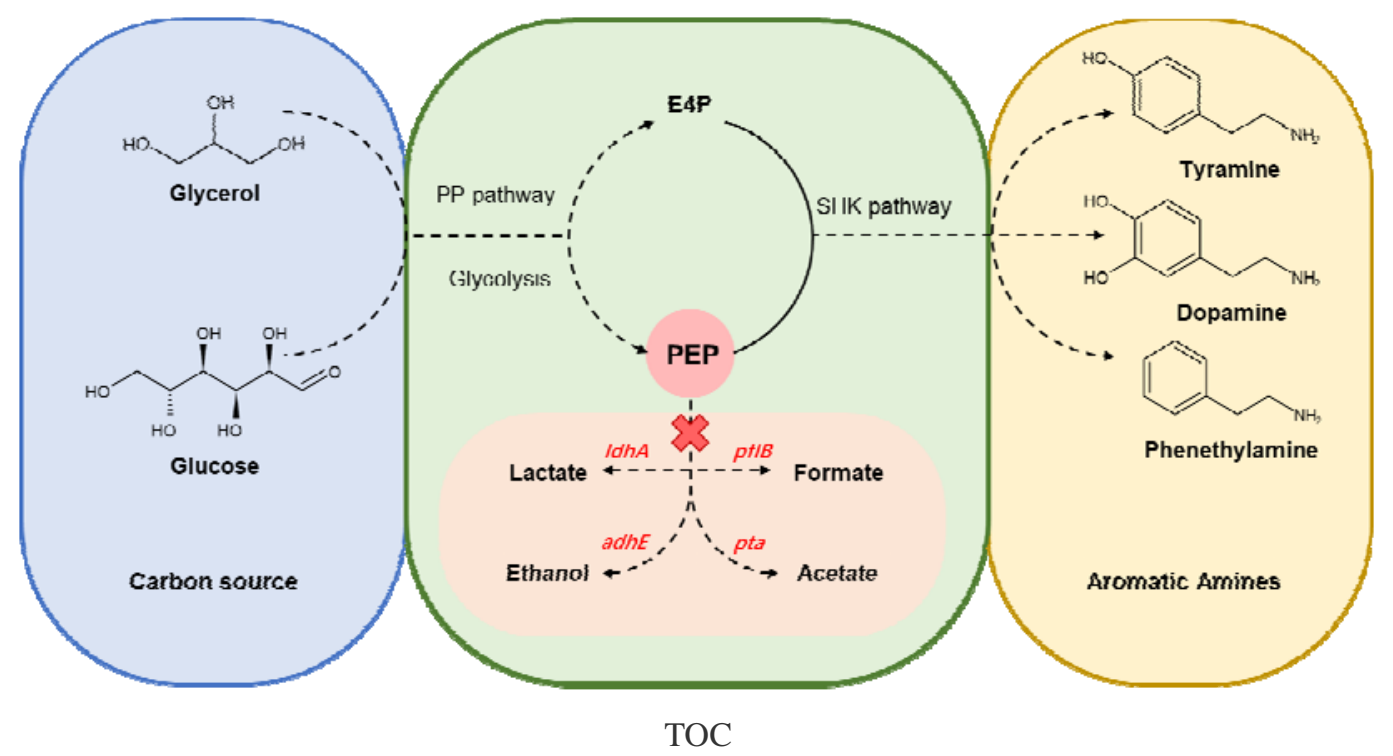

464 